\title{
Electromagnetic dipole radiation of oscillating D-branes
}

\author{
G.K.Savvidy ${ }^{\mathrm{a}}$

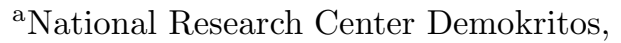 \\ Ag. Paraskevi, GR-15310 Athens,Greece
}

I emphasize analogy between Dp-branes in string theories and solitons in gauge theories comparing their common properties and showing differences. In string theory we do not have the full set of equations which define the theory in all orders of coupling constant as it was in gauge theories, nevertheless such solutions have been found as solutions of low energy superstring effective action carrying the RR charges. The existence of dynamical RR charged extended objects in string theory has been deduced also by considering string theory with mixed boundary conditions, when type II closed supersting theory is enriched by open strings with Neumann boundary conditions on $p+1$ directions and Dirichlet conditions on the remaining 9-p transverse directions.

We will show that for certain excitations of the string/D3-brane system Neumann boundary conditions emerge from the Born-Infeld dynamics. Here the excitations which are coming down the string with a polarization along a direction parallel to the brane are almost completely reflected just as in the case of all-normal Dirichlet excitations considered by Callan and Maldacena, but now the end of the string moves freely on the 3-brane realizing Polchinski's open string Neumann boundary condition dynamically.

In the low energy limit $\omega \rightarrow 0$, i.e. for wavelengths much larger than the string scale only a small fraction $\sim \omega^{4}$ of the energy escapes in the form of dipole radiation. The physical interpretation is that a string attached to the 3-brane manifests itself as an electric charge, and waves on the string cause the end point of the string to freely oscillate and produce e.m. dipole radiation in the asymptotic outer region. The magnitude of emitted power is in fact exactly equal to the one given by Thompson formula in electrodynamics.

\section{INTRODUCTION}

Probably the best way to introduce D-branes is to recall that in non-Abelian gauge field theories there exist classical BPS solutions corresponding to new particles, extended objects, vortices and monopoles which are not present in the perturbative spectrum of the quantized theory [1-3]. In these theories in addition to the point particle spectrum of perturbation theory consisting of a massless photon, massive vector bosons and Xiggs particles there also exist stable solutions with masses inversely proportional to the square of the coupling constant [1-3]

$(\text { Mass })_{B P S}^{2}=4 \pi M_{W} / e^{2}$.

where $M_{W}$ is a vector boson mass. In the case of vortices it is energy per unit of length and should be associated with the string tension. These states cannot be seen in the weak coupling limit simply because the masses of these states become very large when $e \rightarrow 0$, therefore in gauge theo- ries we have mass hierarchy: the particle masses are of order $O\left(e^{0}\right)$ and the soliton masses are of order $O\left(1 / e^{2}\right)$. These states are invisible in perturbation theory. One can probably think about these solutions as "typhoons" of gauge bosons.

The second important message which bring to us these solutions is that extended objects in gauge theories carry a new type of charge, in the given case it is magnetic charge [2], which was not present in perturbative spectrum, as well

$g=2 \pi / e$.

The magnetic charge satisfies the Dirac quantization condition

$e g=2 \pi n \quad n \in Z$,

which ties together the units of electric and magnetic charges $[4,5]$. (Generally speaking magnetic charge is realized as a genuine solution without singularities when $U(1)$ is embedded into nonAbelian compact group [2].) With the existence of magnetic charges and also dyons - particles 
carrying electric and magnetic charges [3] the theory may exhibit full electromagnetic duality [6-8]. According to this conjecture, the strong coupling limit of the theory is equivalent to the weak coupling limit with ordinary particles and solitons exchanged.

In string theory one can also expect solutions which cannot be seen in the perturbation theory. In string theory we do not have the full set of equations which define the theory to all orders of coupling constant as it was in gauge theories, nevertheless such solutions have been found as solutions of low energy superstring effective action [9]. The lagrangian can be represented in the form:

$$
\begin{array}{r}
S=\frac{1}{16 \pi G_{N}^{(10)}} \int d^{10} x \sqrt{-g} \\
\left\{e^{-2 \varphi}\left[R+4(\partial \varphi)^{2}-\frac{1}{3} H^{2}\right]-\frac{1}{3} G^{2}\right\}
\end{array}
$$

where $\varphi$ is the dilaton field, $H$ - the NS-NS gauge field and $G$ - RR gauge field have essentially different order in dilaton coupling for RR fields. The corresponding equations have two types of solutions carrying NS-NS or RR charges [9].

The NS-NS soliton is determined by the balance between the first three terms and its tension (tension $\equiv$ mass/volume ), scales as the action (4)

$(\text { Tension })_{N S-N S} \simeq \frac{1}{g_{s}^{2}}$

where $e^{-\varphi}=g_{s}$ is the string coupling constant. In NS-NS sector the electrically charged object is the fundamental string of the mass $O\left(g_{s}^{0}\right)$ and is accompanied by the above solitonic D5-brane carring the dual magnetic charge of the mass $O\left(1 / g_{s}^{2}\right)$. This is very similar to what we had in gauge theories.

In $\mathrm{RR}$ sector the situation is different, all string states are neutral with respect to $R R$ gauge field and in perturbative string spectrum there is no fundamental objects carrying elementary RR charges $[10,11]$. In the $\mathrm{RR}$ sector the solution of the equations is determined by the balance between the first three and the fourth term in the effective action (4) and the tension of the solution scales as

$(\text { Tension })_{R R} \simeq \frac{1}{g_{s}}$

so that both types of RR charges, electric p-brane and its dual magnetic 6 -p brane, are solitons of the mass $O\left(1 / g_{s}\right)$ which is smaller than that of the NS-NS solution (5). This behaviour, intermediate between fundamental string spectrum $O\left(g_{s}^{0}\right)$ and solitonic brane $O\left(1 / g_{s}^{2}\right)$, was not known in field theory (see Figure 3 ).

The existence of dynamical extended objects in string theory which carry RR charges can be deduced also by considering string theory with mixed boundary conditions [10]. Indeed one can consider type II closed superstring theory which has been completed by adding open strings with Neumann boundary conditions on $p+1$ directions and Dirichlet conditions on the remaining 9-p transverse directions $[10,11,13,14]$,

$n^{a} \partial_{a} X^{\mu}=0, \quad \mu=0, \ldots p$,
$X^{\mu}=0, \quad \mu=p+1, \ldots 9$.

Here the open string endpoints live on the extended object, D-brane, and in this indirect way defines a string soliton with $p$ spatial and one timelike coordinates. The D-branes are surfaces of dimension $\mathrm{p}$ on which open string can end. This is a consistent string theory if $p$ is even in type IIA theory and is odd in type IIB theory [10]. The identification with solitons can be seen if one compute the tension $[10,11]$

$\tau_{p}=\frac{1}{g_{s}(2 \pi)^{\frac{p-1}{2}}\left(2 \pi \alpha^{\prime}\right)^{\frac{p+1}{2}}}$

This is the same coupling constant dependence as for the branes carrying RR charges (6)! The RR charge which carry D-brane is

$\mu_{p}=\frac{1}{(2 \pi)^{-\frac{1}{2}}\left(4 \pi^{2} \alpha^{\prime}\right)^{\frac{p-3}{2}}}$.

and satisfies the Dirac quantization condition

$\mu_{p} \mu_{6-p}=2 \pi n \quad n \in Z$

with $n=1$. Thus RR gauge fields naturally couple to Dp-branes and identify Dp-branes as carrier of RR charge [10]. The general remark 
is that the tension of the p-brane should have dimension $\mathrm{mass}^{p+1}$ and it is indeed so in (9), but what is important is that it is a function of the fundamental string tension $1 / 2 \pi \alpha^{\prime}$ (as well as $\left.1 / g_{s}\right)$ and not just a new parameter of the theory $[15,16,18]$. The D-branes are indeed the "typhoons" of strings.

\section{THE D-BRANE EXCITATIONS}

The Dp-brane is a dynamical extended object which can fluctuate in shape and position and these fluctuations are described by the attached open strings. The boundary condition (8) allows open string ends to be at any place inside the p-brane with coordinates $x^{0}, \ldots, x^{p}$ and $x^{p+1}=\ldots=x^{9}=0$. In this way the open string describes the excitations of the Dp-brane, and as it is well known the open string massless states are vector and spinor fields of ten-dimensional $N=1$ supersymmetric $U(1)$ gauge theory in ten dimensions. The massless field $A_{\mu}\left(x^{\nu}\right), \mu, \nu=0, \ldots, p$ propagates as gauge bosons on the p-brane worldvolume, while the other components of the vector potential $A_{4}\left(x^{\nu}\right), \ldots, A_{9}\left(x^{\nu}\right)$ can be interpreted as oscillation in the position of the p-brane. Thus the theory on the p-brane worldvolume is a tendimensional supersymmetric $U(1)$ gauge theory dimensionally reduced to $p+1$ dimensions. The low energy effective action for these fields is the Dirac-Born-Infeld lagrangian $[12,31]$. The key difference with previously known p-branes and supermembranes [17] is the inclusion of a worldvolume electromagnetic field.

Callan and Maldacena [20] showed that the Dirac-Born-Infeld action, can be used to build a configuration with a semi-infinite fundamental string ending on a 3 -brane ${ }^{1}$, whereby the string is actually made out of the brane wrapped on $S_{2}$ (see also [21]). The relevant action can be obtained by computing a simple Born-Infeld determinant, dimensionally reduced from 10 dimen-

\footnotetext{
${ }^{1}$ We review the favorite case of the 3-brane first because of its non-singular behaviour in SUGRA, and secondly it is suggestive of our own world which is after all 3dimensional.
}

sions

$L=-\frac{1}{(2 \pi)^{3} g_{s}} \int d^{4} x \sqrt{1-\vec{E}^{2}+\left(\vec{\partial} x_{9}\right)^{2}}$,

where $g_{s}$ is the string coupling $\left(\alpha^{\prime}=1\right)$.

The above mentioned theory contains 6 scalars $x_{4}, \ldots, x_{9}$, which are essentially Kaluza-Klein remnants from the 10-dimensional $N=1$ electrodynamics after dimensional reduction to $3+1$ dimensions. As we explain these extra components of the e.m. field $A_{4}, \ldots, A_{9}$ describe the transverse deviations of the brane $x_{4}, \ldots, x_{9}$.

The solution, which satisfies the BPS conditions, is necessarily also a solution of the linear theory, the $N=4$ super-Electrodynamics [20],

$\vec{E}=\frac{c}{r^{2}} \overrightarrow{e_{r}}, \quad \vec{\partial} x_{9}=\frac{c}{r^{2}} \overrightarrow{e_{r}}, \quad x_{9}=-\frac{c}{r}$,

where $c=2 \pi^{2} g_{s}$ is the unit charge, and can be thought of as setting the distance scale. Here the scalar field represents the geometrical spike, and the electric field insures that the string carries uniform NS charge along it. The RR charge of the 3-brane [19]

$\Omega^{M N L}=\epsilon^{a b c} \partial_{a} X^{M} \partial_{b} X^{N} \partial_{c} X^{L}$

cancels out on the string, or rather the tube behaves as a kind of RR dipole whose magnitude can be ignored when the tube becomes thin. It was demonstrated in [20] that the infinite electrostatic energy of the point charge can be reinterpreted as being due to the infinite length of the attached string. The energy per unit length comes from the electric field and corresponds exactly to the fundamental string tension.

Polchinski, when he introduced D-branes as objects on which strings can end, required that the string has Dirichlet (fixed) boundary conditions for coordinates normal to the brane (8), and Neumann (free) boundary conditions for coordinate directions parallel to the brane (7) [10-12].

It was shown in [20] that small fluctuations which are normal to both the string and the brane are mostly reflected back with a phase shift $\rightarrow \pi$ which indeed corresponds to Dirichlet boundary condition (8). See also [32] and [30] for a supergravity treatment of this problem.

In the paper [23] it was demonstrated that $\mathrm{P}$-wave excitations which are coming down the 
string with a polarization along a direction parallel to the brane are almost completely reflected just as in the case of all-normal excitations, but the end of the string moves freely on the 3-brane, thus realizing Polchinski's open string Neumann boundary condition (7) dynamically. As we will see a superposition of excitations of the scalar $x_{9}$ and of the electromagnetic field reproduces the required behaviour, e.g. reflection of the geometrical fluctuation with a phase shift $\rightarrow 0$ (Neumann boundary condition).

In [23] it was also observed the electromagnetic dipole radiation which escapes to infinity from the place where the string is attached to the 3-brane. It was shown that in the low energy limit $\omega \rightarrow 0$, i.e. for wavelengths much larger than the string scale a small fraction $\sim \omega^{4}$ of the energy escapes to infinity in the form of electromagnetic dipole radiation. The physical interpretation is that a string attached to the 3-brane manifests itself as an electric charge, and waves on the string cause the end point of the string to freely oscillate and produce electromagnetic dipole radiation in the asymptotic outer region of the 3-brane. This result is also in a good agreement with the interpretation of the open string ending on D-brane (7), according to which the open string states describe the excitations of the D-brane. Indeed as we will see open string massless vector boson can propagate inside the D-brane and in our case this excitation is identified with electromagnetic dipole radiation. Thus not only in the static case, but also in a more general dynamical situation the above interpretation remains valid. This result provides additional support to the idea that the electron (quark) may be understood as the end of a fundamental string ending on a D-brane.

\section{THE LAGRANGIAN AND THE E- QUATIONS}

Let us write out the full Lagrangian which contains both electric and magnetic fields, plus the scalar $x_{9} \equiv \phi[23]$

$$
L=-\int d^{4} x \sqrt{D e t}
$$

where Det $=1+\vec{B}^{2}-\vec{E}^{2}-(\vec{E} \cdot \vec{B})^{2}$

$$
\begin{array}{r}
-\left(\partial_{0} \phi\right)^{2}\left(1+\vec{B}^{2}\right)+(\vec{\partial} \phi)^{2}+(\vec{B} \cdot \vec{\partial} \phi)^{2} \\
-(\vec{E} \times \vec{\partial} \phi)^{2}+2 \partial_{0} \phi(\vec{B}[\vec{\partial} \phi \times \vec{E}])
\end{array}
$$

We will proceed by adding a fluctuation to the background values (13) :

$$
\vec{E}=\vec{E}_{0}+\delta \vec{E}, \quad \vec{B}=\delta \vec{B}, \quad \phi=\phi_{0}+\eta .
$$

Then keeping only terms in the Det which are linear and quadratic in the fluctuation we will get

$$
\begin{gathered}
\delta \text { Det }=\delta \vec{B}^{2}-\delta \vec{E}^{2}-\left(\vec{E}_{0} \delta \vec{B}\right)^{2}-\left(\partial_{0} \eta\right)^{2}(16) \\
+(\vec{\partial} \eta)^{2}+(\delta \vec{B} \vec{\partial} \phi)^{2}-\left(\vec{E}_{0} \times \vec{\partial} \eta\right)^{2}-(\delta \vec{E} \times \vec{\partial} \phi)^{2} \\
-2\left(\vec{E}_{0} \times \vec{\partial} \eta\right)(\delta \vec{E} \times \vec{\partial} \phi)-2\left(\overrightarrow{E_{0}} \delta \vec{E}\right)+2(\vec{\partial} \phi \vec{\partial} \eta)
\end{gathered}
$$

Note that one should keep the last two linear terms because they produce additional quadratic terms after taking the square root. These terms involve the longitudinal polarization of the e.m. field and cancel out at quadratic order. The resulting quadratic Lagrangian is [23]

$$
\begin{aligned}
2 L_{q} & =\delta \vec{E}^{2}\left(1+(\vec{\partial} \phi)^{2}\right)-\delta \vec{B}^{2}+\left(\partial_{0} \eta\right)^{2} \\
& -(\vec{\partial} \eta)^{2}\left(1-{\overrightarrow{E_{0}}}^{2}\right)+{\overrightarrow{E_{0}}}^{2}(\vec{\partial} \eta \cdot \delta \vec{E}) .
\end{aligned}
$$

Let us introduce the gauge potential for the fluctuation part of the e.m. field as $\left(A_{0}, \vec{A}\right)$ and substitute the values of the background fields from (13)

$$
\begin{array}{r}
2 L_{q}=\left(\partial_{0} \vec{A}-\vec{\partial} A_{0}\right)^{2}\left(1+\frac{1}{r^{4}}\right)-(\vec{\nabla} \times \vec{A})^{2} \\
+\left(\partial_{0} \eta\right)^{2}-(\vec{\partial} \eta)^{2}\left(1-\frac{1}{r^{4}}\right)+\frac{1}{r^{4}}\left(\partial_{0} \vec{A}-\vec{\partial} A_{0}\right) \cdot \vec{\partial} \eta .
\end{array}
$$

The equations that follow from this lagrangian contain dynamical equations for the vector potential and for the scalar field, and a separate equation which represents a constraint. These equations in the Lorenz gauge $\vec{\partial} \vec{A}=\partial_{0} A_{0}$ are

$$
\begin{array}{r}
-\partial_{0}^{2} \vec{A}\left(1+\frac{1}{r^{4}}\right)+\Delta \vec{A}+\frac{1}{r^{4}} \vec{\partial} \partial_{0}\left(A_{0}+\eta\right)=0 \\
-\partial_{0}^{2} A_{0}+\Delta A_{0}+\vec{\partial} \frac{1}{r^{4}} \vec{\partial}\left(A_{0}+\eta\right)-\vec{\partial} \frac{1}{r^{4}} \partial_{0} \vec{A}=0 \\
-\partial_{0}^{2} \eta+\Delta \eta-\vec{\partial} \frac{1}{r^{4}} \vec{\partial}\left(A_{0}+\eta\right)+\vec{\partial} \frac{1}{r^{4}} \partial_{0} \vec{A}=0
\end{array}
$$

Equation (20) is a constraint: the time derivative of the lhs is zero, as can be shown using the equation of motion (19). 
Let us choose $A_{0}=-\eta$. This condition can be viewed as (an attempt to) preserve the BPS relation which holds for the background: $\vec{E}=\vec{\partial} \phi$. Another point of view is that this fixes the general coordinate invariance which is inherent in the Born-Infeld lagrangian in such a way as to make the given perturbation to be normal to the surface. Of course transversality is insured automatically but this choice makes it explicite. The general treatment of this subject can be found in [31].

With this condition the equations (20) and (21) become the same, and the first equation is also simplified [23]:

$$
\begin{aligned}
& -\partial_{0}^{2} \vec{A}\left(1+\frac{1}{r^{4}}\right)+\Delta \vec{A}=0, \\
& -\partial_{0}^{2} \eta+\Delta \eta+\vec{\partial} \frac{1}{r^{4}} \partial_{0} \vec{A}=0 .
\end{aligned}
$$

This should be understood to imply that once we obtain a solution, $A_{0}$ is determined from $\eta$, but in addition we are now obliged to respect the gauge condition which goes over to $\vec{\partial} \vec{A}=-\partial_{0} \eta$.

\section{NEUMANN BOUNDARY CONDITI- ONS FROM BORN-INFELD DYNAM- ICS [23]}

We will seek a solution with definite energy (frequency $w$ ) in the following form: $\vec{A}$ should have only one component $A_{z}$, and $\eta$ be an $l=1$ spherical $P$-wave

$$
A_{z}=\zeta(r) e^{-i \omega t}, \quad \eta=\frac{z}{r} \psi(r) e^{-i \omega t}
$$

The geometrical meaning of such a choice for $\eta$ is explained in Figure 1, and the particular choice of $z$ dependence corresponds to the polarization of the oscillations along the $z$ direction of the brane. With this ansatz the equations become

$$
\begin{aligned}
&\left(1+\frac{c^{2}}{r^{4}}\right) \omega^{2} \zeta+\frac{c^{2}}{r^{2}} \partial_{r}\left(r^{2} \partial_{r} \zeta\right)=0, \\
& \frac{z}{r} \omega^{2} \psi+\frac{z}{r} \frac{1}{r^{2}} \partial_{r}\left(r^{2} \partial_{r} \psi\right)+\frac{z}{r} \frac{2}{r^{2}} \psi- \\
& i \omega \partial_{z}\left(\frac{c^{2}}{r^{4}} \zeta\right)=0,
\end{aligned}
$$

with the gauge condition becoming $\partial_{r} \zeta=i \omega \psi$. It can be seen again, that the second equation
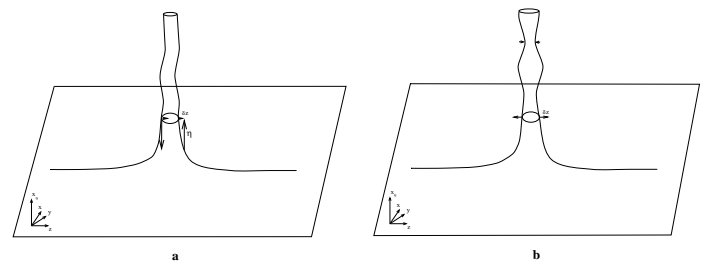

Figure 1. In order that all points on the $S_{2}$ section of the tube ( which is schematically shown here as a circle) move all in the same direction $\hat{z}$ by an equal distance $\delta z$, the field $\eta$ has to take on different values at, say, opposite points of the $S_{2}$. In effect, $\eta=\delta \frac{-1}{r}=\frac{1}{r^{2}} \frac{z}{r} \delta z$. If, on the other hand, it is taken to be an $S$-wave (as in the paper [30]) that would correspond to Fig 1b, which at best is a problematic 'internal' degree of freedom of the tube.

follows from the first by differentiation. This is because the former coincides with the constraint in our ansatz.

Therefore the problem is reduced to finding the solution of a single scalar equation, and determining the other fields through subsidiary conditions. The equation (24) itself surprisingly turned out to be the one familiar from [20] for the transverse fluctuations. There it was solved by going over to a coordinate $\xi$ which measures the distance radially along the surface of the brane

$$
\xi(r)=\omega \int_{\sqrt{c}}^{r} d u \sqrt{1+\frac{c^{2}}{u^{4}}},
$$

and a new wavefunction

$$
\tilde{\zeta}=\zeta\left(c^{2}+r^{4}\right)^{1 / 4} .
$$

This coordinate behaves as $\xi \sim r$ in the outer region $(r \rightarrow \infty)$ and $\xi \sim-1 / r$ on the string $(r \rightarrow$ $0)$. The exact symmetry of the equation $r \leftrightarrow$ $c / r$ goes over to $\xi \leftrightarrow-\xi$. The equation, when written in this coordinate becomes just the free wave equation, plus a narrow symmetric potential at $\xi \sim 0$

$$
-\frac{d^{2}}{d \xi^{2}} \tilde{\zeta}+\frac{5 c^{2} / \omega^{2}}{\left(r^{2}+c^{2} / r^{2}\right)^{3}} \tilde{\zeta}=\tilde{\zeta} .
$$


The asymptotic wave functions can be constructed as plain waves in $\xi$,

$$
\zeta(r)=\left(c^{2}+r^{4}\right)^{-1 / 4} e^{ \pm i \xi(r)},
$$

or in the various limits:

$$
\begin{array}{r}
r \rightarrow 0 \quad \zeta \sim \frac{1}{\sqrt{c}} e^{ \pm i \xi(r)}, \\
r \rightarrow \infty \quad \zeta \sim \frac{1}{r} e^{ \pm i \xi(r)} .
\end{array}
$$

These formulae give us the asymptotic wave function in the regions $\xi \rightarrow \pm \infty$, while around $\xi=$ $0(r=1)$ there is a symmetric repulsive potential which drops very fast $\sim 1 / \xi^{6}$ on either side of the origin. The scattering is described by a single dimensionless parameter $\omega \sqrt{c}$, and in the limit of small $\omega$ and/or coupling $c=2 \pi^{2} g_{s}$ the potential becomes narrow and high, and can be replaced by a $\delta$-function with an equivalent area $\sim \frac{1}{\omega \sqrt{g_{s}}}$ under the curve. Therefore the scattering matrix becomes almost independent of the exact form of the potential. We refer the reader to pp 18-20 of the thesis [22] for the more detailed calculation of transmission amplitude (29). The end result is that most of the $\zeta$ amplitude is reflected back with a phase shift close to $\pi$.

In order to obtain $\psi$ (and $\eta$ ) we need to differentiate $\zeta$ with respect to $r$ :

$$
\begin{array}{r}
i \omega \psi=\frac{-1}{4} \frac{4 r^{3}}{\left(c^{2}+r^{4}\right)^{5 / 4}} e^{ \pm i \xi(r)} \\
\pm \frac{i \omega}{\left(c^{2}+r^{4}\right)^{1 / 4}}\left(1+\frac{c^{2}}{r^{4}}\right)^{1 / 2} e^{ \pm i \xi(r)} .
\end{array}
$$

Again it is easy to obtain the simplified limiting form:

$$
\begin{array}{r}
r \rightarrow 0 \quad i \omega \psi \sim\left(-\frac{r^{3}}{c^{5 / 2}} \pm \frac{i \omega \sqrt{c}}{r^{2}}\right) e^{ \pm i \xi(r)} \\
r \rightarrow \infty \quad i \omega \psi \sim\left(\frac{-1}{r^{2}} \pm \frac{i \omega}{r}\right) e^{ \pm i \xi(r)}
\end{array}
$$

This brings about several consequences for $\psi$. Firstly, it causes $\psi$ to grow as $\sim 1 / r^{2}$ when $r \rightarrow 0$. This is the correct behaviour because when converted to displacement in the $z$ direction, it means constant amplitude [23]:

$$
\eta=\frac{z}{r} \psi=\delta \frac{-c}{r}=\frac{z}{r} \frac{c}{r^{2}} \delta z \Rightarrow \delta z \sim \text { const } .
$$

Secondly, the $i$ that enters causes the superposition of the incoming and reflected waves to become a cosine from a sine, as is the case for $\zeta$ waves. This corresponds to a 0 phase shift for the $\eta$ wave (Figure 2) and implies therefore Neumann boundary condition, that is it allows open string ends to be at any place inside the p-brane.

\section{ELECTROMAGNETIC DIPOLE RA- DIATION OF OSCILLATING D- BRANE [23]}

Because of the $\omega$ factor in the gauge condition we need to be careful about normalizations, thus we shall choose to fix the amplitude of the $\delta z$ (or $\eta$ ) wave to be independent of frequency $\omega$. Then the magnitude of the electromagnetic field in the inner region becomes independent of $\omega$ as well, thus the amplitude of $\zeta$ wave at $r \rightarrow 0$ is

$$
A_{z}=\zeta_{0} e^{-i \omega(t+c / r)}
$$

Combined with the transmission factor

$T=-i \omega \sqrt{c}+O\left(\omega^{2}\right)$,

this gives the following form of the dipole radiation field at infinity ${ }^{2}$

$$
\begin{aligned}
A_{z}=\frac{T \cdot \zeta_{0}}{r} e^{-i \omega t} & =\frac{-i \omega \sqrt{2 \pi^{2} g_{s}}}{r} \zeta_{0} e^{-i \omega t} \\
& =\frac{-i \omega e \zeta_{0}}{r} e^{-i \omega t}=\frac{\dot{d}}{r}
\end{aligned}
$$

where

$$
d=e \zeta_{0} e^{-i \omega t}
$$

In order to make a comparison with Thompson formula

$$
I=\frac{4}{3} \omega^{4} e^{2} \zeta_{0}{ }^{2}
$$

for the total power emitted by an oscillating dipole, we note that the agreement is guaranteed if the exact coefficient behind $-i \omega \sqrt{c}$ in (29) is equal to one. As it was shown in [22], the approximate computation of the transmission amplitude $T$ (29) gives $T \sim-i .92 \omega \sqrt{c}$. The later

\footnotetext{
The unit of electric charge in our notation is $2 \pi^{2} g_{s}=e^{2}$. This is because of the scaling of the fields needed to get the $\mathrm{U}(1)$ action with $1 / e^{2}$ in front of it.
} 


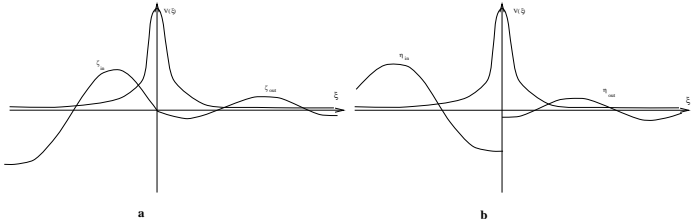

Figure 2. The figure 2a depicts the scattering of the $\zeta$ wave. Note the discontinuity in the derivative which is proportional to $1 / \omega$. Figure $2 \mathrm{~b}$ shows the scattering of the $\eta$ wave. Being the derivative of $\zeta$, it results in a discontinuity of the function itself, making it into a cosine, which means free (Neumann) boundary condition at $\xi=0$.

computation of this coefficient using the numerical solution of the equation (24) shows that it is indeed equal to one. To solve properly the equation on a computer it is necessary to introduce new variables [24]

$y=r-\frac{c}{r}$, or $\quad r(y)=\frac{y+\sqrt{y^{2}+4 c}}{2}$,

so that the equation (24) take the form

$\omega^{2}\left(c^{2}+r(y)^{4}\right) \zeta+\left(c+r(y)^{2}\right) \partial_{y}\left(c+r(y)^{2}\right) \partial_{y} \zeta=0(32)$

and boundary condition at $r_{0}=\omega c / 2 \pi n, n \rightarrow \infty$ the form

$$
\begin{array}{r}
\zeta\left(y_{0}\right)=\frac{1}{\sqrt{c}}, \quad \zeta^{\prime}\left(y_{0}\right)=-i \frac{(2 \pi n)^{2}}{\omega c^{3 / 2}}, \\
\text { at } \quad y_{0}=\frac{\omega c}{2 \pi n}-\frac{2 \pi n}{\omega} \rightarrow-\infty .
\end{array}
$$

Measuring the amplitudes $y \zeta(y) \pm\left(y+\frac{\pi}{2 \omega}\right) \zeta(y+$ $\left.\frac{\pi}{2 \omega}\right)$ at $y \rightarrow \infty(r \rightarrow \infty)$ one can get that the coefficient behind $-i \omega \sqrt{c}$ in (29) is equal to one. Thus the magnitude of emitted power is in fact exactly equal to the one given by Thompson formula in electrodynamics ${ }^{3}$.

In conclusion, we need to analyze the outgoing scalar wave. This wave has both real and imaginary parts, the imaginary part is $\sim 1 / r^{2}$ and drops faster than radiation. The real part does

${ }^{3}$ see also [25-29]
$O\left(1 / g^{2}\right)$

$O(1 / g)$

$\mathrm{O}\left(\mathrm{g}^{0}\right)$

Figure 3. In NS-NS sector the electrically charged object is the fundamental string of the mass $O\left(g_{s}^{0}\right)$ and is accompanied by the solitonic D5-brane carring the dual magnetic charge of the mass $O\left(1 / g_{s}^{2}\right)$. In the RR sector both types of RR charges, electric p-brane and its dual magnetic $6-p$ brane, are solitons of the mass $O\left(1 / g_{s}\right)$ which is smaller than that of the NS-NS solution. This behaviour, intermediate between fundamental string spectrum $O\left(g_{s}^{0}\right)$ and solitonic brane $O\left(1 / g_{s}^{2}\right)$, was not known in field theory.

contribute to the radiation at spatial infinity, as can be shown from the integral of the energy density $\int\left(\partial_{r} \eta\right)^{2} d^{3} r \sim \int \omega^{4} / r^{2} \cdot 4 \pi r^{2} d r \sim \omega^{4}$. This is not altogether surprising, as we are dealing with a supersymmetric theory where the different fields are tied together. In fact, it is easy to show that the fields still do asymptotically satisfy the BPS conditions at infinity, and $1 / 4$ of the SUSY is preserved. Thus the observer at spatial infinity will see both an electromagnetic dipole radiation field and a scalar wave. Interestingly, the direction dependences of the two conspire to produce a spherically symmetric distribution of the energy radiated.

I wish to thank the organizers of the conference QG99 in Villasimius and especially Vittorio de Alfaro for the invitation and for arranging interesting and stimulating meeting. I also thank Konstantin Savvidy for the enjoyable collaboration on the work reported here. The work was supported in part by the EEC Grant no. HPRNCT-1999-00161 and ESF Grant "Geometry and Disorder: From Membranes to Quantum Grav- 
ity".

\section{REFERENCES}

1. H.B. Nielsen and P. Olesen, Nucl. Phys. B61 (1973) 45.

2. G.t́ Hooft Nucl. Phys. B79 (1974) 276; A. Polyakov, JETP Lett. 20 (1974) 194.

3. B. Julia and A. Zee, Phys. Rev. D11 (1975) 2227.

4. P.A.M. Dirac, Proc. Roy. Soc. A133 (1934) 60.

5. J. Schwinger, Phys. Rev. 144 (1966) 1087.

6. C. Montonen and D. Olive, Phys. Lett. 72B (1977) 117.

7. E. Witten and D. Olive, Phys. Lett. 78B (1978) 97.

8. N. Seiberg and E. Witten, Nucl. Phys. B426 (1994) 19.

9. G.T. Horowitz and A. Strominger, Nucl. Phys. B360 (1991) 197; A. Dabholkar, G. Gibbons, J. Harvey and F. Ruiz Ruiz, Nucl. Phys. B340 (1990) 33; S.M. Hull and P.K. Townsend, Nucl. Phys. B438 (1995) 109; E. Witten, Nucl. Phys. B443 (1995) 85; A. Strominger, Nucl. Phys. B451 (1995) 85; K. Stelle, Lectures on Supergravity p-branes, hep-th/9710046; M.J. Duff, R.R. Khuri and J.X. Lu, String solitons, Phys. Rep. 259 (1995) 213-326.

10. J. Polchinski, Phys. Rev. Lett. 75 (1995) 4724, hep-th/9510017; J. Polchinski, Tasi lectures on D-branes, hep-th/9611050.

11. J. Dai, R.G. Leigh, J. Polchinski, Mod. Phys. Lett. A4 (1989) 2073.

12. R.G. Leigh, Mod. Phys. Lett. A4 (1989) 2767.

13. C. Bachas, (Half) a lecture on D-branes, hepth/9701019.

14. E. Kiritsis, Supersymmetry and Duality in Field Theory and String Theory, hep$\mathrm{ph} / 9911525$.

15. G. Baseyan, S. Matinyan and G. Savvidy, Pisma Zh. Eksp. Teor. Fiz. 29 (1979) 641; G. Savvidy, Nucl. Phys. B246 (1984) 302.

16. M.E. Laziev and G.K. Savvidy, Phys. Lett. B198 (1987) 451; Sov. J. Nucl. Phys. 47 (1988) 304; G.K. Savvidy, Sov. J. Nucl. Phys. 47 (1988) 748; Preprint-CERN-TH-4779/87,
Jul. 1987, 10pp; T.A. Arakelian and G.K. Savvidy, Phys. Lett. B214 (1988) 350.

17. M.J. Duff, Supermembranes: The first fifteen weeks, Class. Quant. Grav. 5 (1988) 189; Supermembranes, hep-th/9611203.

18. G.K. Savvidy, Symplectic and large-N gauge theories, in NATO ASI Series v. 255 (1991) 415.

19. B. Biran, E. Floratos and G. Savvidy, Phys. Lett. B198 (1987) 329.

20. C.G. Callan and J. Maldacena, Nucl. Phys. B513 (1998) 198, hep-th/9708147.

21. G. Gibbons, Nucl. Phys. B514 (1998) 603, hep-th/9709027.

22. K. Savvidy,Born-Infeld Action in String Theory, PhD Thesis (Princeton University, June 1999) hep-th/9906075.

23. K. Savvidy and G. Savvidy, Neumann boundary conditions from Born-Infeld dynamics, hep-th/9902023, Nucl. Phys. B561 (1999) 117.

24. K. Savvidy, October 1999, unpublished.

25. C.G. Callan and A. Guijosa, Undulating strings and gauge fields, hep-th/9906153.

26. M. Abou-Zeid and M. Costa, Radiation from accelarated branes, hep-th/9909148.

27. C. Bachas and B. Pioline. High energy scattering on distant branes, hep-th/9909171.

28. R. Janik and R. Peschanski, High energy scattering and the AdS/CFT correpondance, hepth/9907177.

29. M. Rho, S-J. Sin and I. Zahed, Elastic Parton-Parton scattering from AdS/CFT, hep-th/9907126.

30. S.-J. Rey, J.-T. Lee, hep-th/9803001.

31. M. Aganagic, C. Popescu and J.H. Schwarz, Nucl. Phys. B495 (1997) 99, hep-th/9612080.

32. S. Lee, A. Peet and L. Thorlacius, Nucl. Phys. 514 (1998) 161, hep-th/9710097.

33. C.G. Callan and L. Thorlacius, Nucl. Phys. B534 (1998) 121, hep-th/9803097. 